\title{
ADRENAL MASSES-A BLEEDING PROBLEM
}

You-Jin Chang, Angela Alina Khan, Sobia Arshad, Ian Scobie.

Department of Endocrinology, Medway Maritime Hospital, Gillingham, Kent

\section{Introduction:}

With the ever-increasing use of CT imaging to achieve surgical diagnoses, endocrinologists are being deluged with referrals of patients found incidentally to have lesions in one or both adrenal glands. Adrenal incidentalomas are defined as clinically unsuspected adrenal masses discovered serendipitously on abdominal imaging (incidence 1-5\% of abdominal CT scans). They are usually associated with no signs of hormonal excess or evidence of obvious underlying malignancy. Around $75-85 \%$ are non-secretory. If hyper-secretory, cortisolsecreting adenomas and phaeochromocytomas predominate. Metastases are very rare in non-oncological patients. The purpose of this report is to present a rare cause of bilateral adrenal enlargement not described above.

\section{Case report:}

A 64 year old female presented with acute right sided abdominal pain shortly after a left knee replacement. She had been started on treatment dose LMWH by her GP for a presumed DVT. The left leg doppler scan was negative for DVT but an abdominal US revealed a solid lesion at the upper pole of the left kidney. CT urography showed large bilateral adrenal masses (image1). No clinical or biochemical evidence of adrenal endocrine excess or deficiency was demonstrated. She became persistently pyrexial during admission, but no source of sepsis was found. Repeat CT scanning with an adrenal washout protocol showed a marked reduction in size of both adrenals, the appearances suggesting resolving bilateral adrenal haemorrhage (BAH) ( image2). A 0900h plasma cortisol level, urinary catecholamine excretion and a plasma aldosterone : renin ratio were all normal.

\section{Discussion:}

The incidence of BAH is estimated to be 4.7-6.2 cases per million (1). Non traumatic adrenal gland haemorrhage is rare. Causes include idiopathic, stress, haemorrhagic coagulopathy or underlying adrenal tumours (2).Adrenal haemorrhage following orthopaedic procedures is well recognised in the literature and may commonly present with abdominal pain and pyrexia $(3,4)$. CT is the recommended imaging modality(5). When adrenal insufficiency complicates BAH immediate treatment with corticosteroids is mandatory (6). We postulate that our patient's bilateral adrenal haemorrhages were a consequence of prior knee surgery although the exhibition of LMWH may have played a contributory or even causative role. There was no substantial evidence to suggest the presence of prior underlying adrenal tumours nor adrenal hyperplasia.

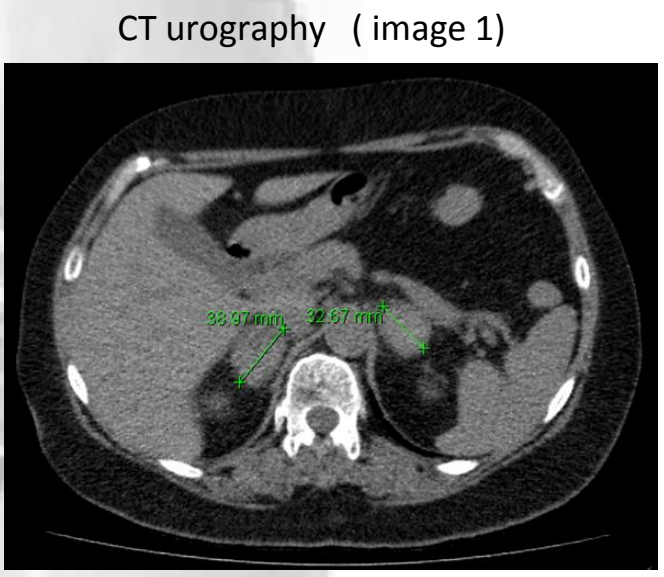

\section{Conclusion}

We draw attention to a rare case of post-operative bilateral adrenal haemorrhage. It underlines the importance of recognising BAH in the differential diagnosis of adrenal masses associated with unexplained abdominal pain and pyrexia since a missed diagnosis could potentially be fatal. 